\title{
Lieferkettengesetz - wie wird es wirksam?
}

\author{
STEFANIE LORENZEN
}

\section{Einführung}

Mehrere Länder haben inzwischen die Verantwortung von Unternehmen für Menschenrechte in Lieferketten gesetzlich geregelt. Meist betreffen sie spezifische Branchen, Themen oder Rechte, etwa ein niederländisches Gesetz gegen Kinderarbeit, Verhaltensstandards zu Konfliktrohstoffen in den USA und der EU, Berichtspflichten in Bezug auf Menschenhandel und moderne Sklaverei in Großbritannien, Australien und den USA. In Deutschland befindet sich im September 2020, zum Entstehungszeitpunkt dieses Beitrags, die Debatte um ein sektorunabhängiges, allgemeines Lieferkettengesetz in einer entscheidenden Phase.

2016 formulierte die Bundesregierung in ihrem Nationalen Aktionsplan Wirtschaft und Menschenrechte (NAP) die Erwartung an alle deutschen Unternehmen, Menschenrechte in ihren Lieferketten zu achten. ${ }^{1}$ Dabei setzte sie grundsätzlich darauf, dass Unternehmen dies freiwillig erfüllen. Bis zum Sommer 2020 wurde in zwei Erhebungsphasen geprüft, ob mindestens die Hälfte der ca. 7200 international aktiven deutschen Unternehmen mit mehr als 500 Arbeitnehmern bereits von sich aus einer menschenrechtlichen Sorgfaltspflicht nachkommen. In der abschließenden Evaluierung erfüllten nur etwa 13-17\% der Unternehmen die Menschenrechtsvorgaben des NAP. Aufgrund dieser mehr als deutlich unter $50 \%$ liegenden Quote sah sich die Bundesregierung aus dem NAP und dem Koalitionsvertrag von 2017 verpflichtet, „national gesetzlich tätig“ zu werden. Federführend für Eckpunkte zu einem Gesetzentwurf waren die Bundesministerien für wirtschaftliche Zusammenarbeit (BMZ), für Arbeit und Soziales (BMAS) und für Wirtschaft (BMWi). Sowohl Entwicklungsminister Gert Müller (CSU) als auch Arbeitsminister Hubertus Heil (SPD) stellten sich wiederholt hinter ein ambitioniertes, haftungsbewehrtes Lieferkettengesetz. Dagegen steht Wirtschaftsminister Peter Altmaier (CDU) dem Projekt insgesamt eher kritisch gegenüber.

Vieles ist in der zum jetzigen Zeitpunkt noch ausstehenden Einigung über Eckpunkte zu einem Gesetz strittig.
Dieser Beitrag diskutiert deshalb, welcher Inhalte und Voraussetzungen es bedarf, um ein deutsches Lieferkettengesetz wirksam machen. Welche Umstände unterstützen, dass die Ziele einer solchen Regulierung erreicht werden? Hierzu werden einige Regelungselemente und Wirkungsbedingungen des Gesetzes selbst untersucht. Darüber hinaus blickt der Beitrag auf weitere Rahmenbedingungen, insbesondere die Rolle der Arbeitnehmervertretungen.

\section{Ziel der Regulierung durch ein Lieferkettengesetz}

Menschenrechtsgefährdungen in globalen Wertschöpfungsketten (GWK) bilden die Ausgangslage. Defizitäre Bedingungen am Arbeitsplatz sowie Umweltverschmutzung und -zerstörung begleiten viele Herstellungsprozesse in GWK. Fabrikbrände oder der Einsatz von Sicherheitskräften gegen Arbeiter zeugen davon. Die Corona-Pandemie hat dies zum Teil zugespitzt, wie die fehlende soziale Sicherung von Millionen entlassener Näherinnen in Asien zeigt. Handelte es sich um rein innerstaatliche Fälle, hätten die Betroffenen in Deutschland sowohl verfahrensrechtlich als auch materiell-rechtlich gute Chancen, ihre Rechte gegen den Arbeitgeber durchzusetzen. In den Produktionsländern, wo der Schaden eintritt, ist dies nicht immer gesichert. Die zum Teil fehlenden oder schlecht funktionierenden rechtsstaatlichen Institutionen und gleichzeitig niedrigen Produktionskosten gelten sogar als wichtiger Outsourcing-Anreiz für Unternehmen der Industriestaaten.

Das Ziel von Regulierung in diesem Bereich müsste dual sein: Es gilt, zum einen Schädigungen von Arbeiter*innen und Gemeinden etwa an Körper, Gesundheit,

\footnotetext{
Nationaler Aktionsplan - Umsetzung der VN-Leitprinzipien für Wirtschaft und Menschenrechte vom 21.12. 2016 (NAP), https://www.bundesregierung.de/breg-de/service/publika tionen/nationaler-aktionsplan-wirtschaft-und-menschen rechte-735164 (letzter Zugriff: 23.09. 2020).
} 
Leben oder Nutzungsrechten zu vermeiden und gegebenenfalls Wiedergutmachung zu ermöglichen, zum anderen reales soziales Upgrading zu erreichen, und zwar durch Erhöhung von Standards - etwa in Bezug auf existenzsichernde Löhne und Kürzung von Überstunden -, aber auch durch verbesserte Ausübungsmöglichkeiten kollektiver Rechte wie freier Gewerkschaftsgründungen und -aktivitäten (vgl. dazu auch den Beitrag von Marslev et al. in diesem Heft).

\section{Ausformulierung des Regulierungsziels - UN-Leitprinzipien für Wirtschaft und Menschenrechte}

Grundsätzliche Bedeutung bei der Konzeptualisierung dieses Ziels kommt den UN-Leitprinzipien für Wirtschaft und Menschenrechte zu. Ihre Meriten liegen darin, einen theoretischen Ansatz mit einem entsprechenden Umsetzungsinstrumentarium zu verbinden. Nach dem RuggieFramework der Vereinten Nationen entsteht ein Interessenausgleich zwischen Wirtschaft und Menschenrechten entlang komplexer, globaler Lieferketten über ein DreiSäulen-Modell. Den Staat trifft die Pflicht, Menschenrechte zu schützen („duty to protect“), die Wirtschaft ist verpflichtet, sie zu achten („duty to respect“), und sowohl Staaten als auch Unternehmen sollen Betroffenen Abhilfe garantieren („access to remedy“). Die $41 \mathrm{UN}$-Leitprinzipien, die das Drei-Säulen-Konzept konkretisieren, hat der UN-Menschenrechtsrat 2011 einstimmig verabschiedet. ${ }^{2}$

Zur Implementierung verlangen die UN-Leitprinzipien von Unternehmen dreierlei: eine Grundsatzerklärung zur Achtung der Menschenrechte, menschenrechtliche Sorgfalt (human rights due diligence) als Kernkonzept und angemessene Beschwerdemöglichkeiten samt Wiedergutmachung bei festgestellten Verletzungen von Menschenrechten. ${ }^{3}$

Alle Elemente beziehen sich auf die „international anerkannten Menschenrechte", mindestens solche aus der Internationalen Menschenrechtscharta und der ILO-Erklärung über die grundlegenden Prinzipien und Rechte bei der Arbeit. Umfasst ist damit eine große Bandbreite an Menschenrechten wie die ILO-Kernarbeitsnormen, zudem etwa das Recht auf Leben, Gesundheit, Freiheit von Zwangsarbeit, gerechte und günstige Arbeitsbedingungen oder auf einen angemessenen Lebensstandard.

Die zentrale menschenrechtliche Sorgfaltspflicht samt Abhilfe und Wiedergutmachung formulieren die UNLeitprinzipien als Verhaltensstandard für Unternehmen, als Methode menschenrechtlichen Risikomanagements. Folgende wesentliche Bestandteile sind dabei zu unterscheiden:

- Risikoanalyse: Unternehmen müssen tatsächliche und potenzielle nachteilige Auswirkungen auf Menschenrechte in ihrer Lieferkette ermitteln (Verfahrenspflicht).

- Prävention: Für identifizierte Risiken sind geeignete
Vorkehrungen festzulegen und in Geschäftsabläufe zu integrieren/umzusetzen (Handlungspflicht).

- Abhilfe: Werden Beeinträchtigungen von Menschenrechten festgestellt, sind sie zu beenden bzw. abzumildern und wiedergutzumachen (Handlungspflicht).

- Wirksamkeitskontrolle: Unternehmen müssen regelmäßig prüfen, ob ihre Präventions- und Abhilfemaßnahmen effektiv sind (Verfahrenspflicht).

- Kommunikation: Unternehmen müssen über ihre menschenrechtliche Sorgfaltspflichterfüllung transparent berichten.

Mit den UN-Leitprinzipien ist ein internationaler Standard entstanden, der mit seiner zentralen Aussage, dass Geschäftstätigkeiten von Unternehmen nicht zur Verletzung von Menschenrechten führen dürfen, fast universelle Akzeptanz erreicht hat. Die UN-Leitprinzipien liegen den meisten neueren nationalen Gesetzen in diesem Bereich zugrunde. Die Verhaltenspflicht zu menschenrechtlicher Sorgfalt in einem deutschen Lieferkettengesetz hierauf aufzubauen, macht das Gesetz anschlussfähig. Es erleichtert ausländischen Geschäftspartnern, den deutschen Standard zu verstehen und umzusetzen. Inzwischen hat außerdem Justizkommissar Reynders auf EU-Ebene Gesetzgebung zu menschenrechtlicher Sorgfalt in GWK angekündigt. Es ist zu erwarten, dass die EU ebenfalls den Standard der UN-Leitprinzipien zugrunde legt.

\section{Wichtige Elemente eines wirksamen Lieferkettengesetzes}

\subsection{Transparenz und Teilhabe}

Die gewünschte Prävention menschenrechtlicher Beeinträchtigungen sollen in erster Linie die Unternehmen an der Spitze von GWK selbst durch einen kontinuierlichen Risikovorsorgezyklus vornehmen. Dabei sprechen erfolgreiche Initiativen für Menschenrechte in GWK - wie der Bangladesh Accord - dafür, dass ein guter Teil des Erfolgs auf der (gleichberechtigten) Beteiligung lokaler Arbeitnehmervertreter und (lokaler, nationaler oder globaler) Gewerkschaften beruht.

Bedeutung für Arbeitnehmervertreter in Produktionsländern: Das wiederum liefert Anhaltspunkte dafür, dass es sinnvoll ist, die Rolle von Arbeitnehmervertretern bei der Umsetzung der menschenrechtlichen Sorgfaltspflicht

2 UN-Dok. A/HRC/R.ES/17/4; http://www.ohchr.org/Docu ments/Publications/GuidingPrinciplesBusinessHR_EN.pdf (letzter Zugriff: 23. 09. 2020).

3 Die OECD-Leitsätze für multinationale Unternehmen (2011) sowie die dreigliedrige Grundsatzerklärung über multinationale Unternehmen und Sozialpolitik der Internationalen Arbeitsorganisation (ILO) (2017) integrieren als wichtige institutionelle Standards ebenfalls die menschenrechtliche Sorgfaltspflicht. 
ernst zu nehmen und zu stärken. Zwar erwähnen die UNLeitprinzipien nur an zwei Stellen explizit Gewerkschaften, die Kommentierung stellt indes klar, dass sie an verschiedenen Stellen der menschenrechtlichen Sorgfalt eine Rolle spielen. Bezüglich der Risikoanalyse können Gewerkschaftsvertreter im Rahmen des sozialen Dialogs verlangen, dass Unternehmen prüfen und offenbaren, inwiefern ihre Einkaufsbedingungen Menschenrechte gefährden. Bei vertieften Risikoprüfungen sind sie als Auskunftspersonen einzubeziehen. Sie können eine aktive Rolle bei der Formulierung eines Abhilfemaßnahmenplans einfordern, eine tarifliche Regelung in Bezug auf Beschwerdemechanismen sowie Beteiligung bei Wiedergutmachungsmaßnahmen verlangen. Ferner sollten Gewerkschaftsvertreter beim Wirksamkeitsmonitoring beteiligt werden, das Design von Sozialaudits mitgestalten und die Ergebnisse einsehen können. ${ }^{4}$ Als Grundvoraussetzung, diese Rechte wahrnehmen zu können, müssen die entsprechenden Informationen transparent zugänglich sein. Die ausreichende Beteiligung sollte sich ebenfalls als Dimension bei der (behördlichen, gerichtlichen) Beurteilung ausreichender menschenrechtlicher Sorgfalt wiederfinden.

Bedeutung für Arbeitnehmervertreter in Deutschland: Deutsche Gewerkschaften sind in der Debatte um die UN Leitprinzipien bzw. deren Übersetzung in ein zwingendes Gesetz recht aktiv. Sowohl der DGB als auch die Einzelgewerkschaft ver.di befürworten klar eine haftungsbewehrte gesetzliche Sorgfaltspflicht.

Interessant ist dagegen, dass laut Studien auf betrieblicher Ebene oft noch keine weiterführenden Kenntnisse über die UN-Leitprinzipien vorhanden sind. ${ }^{5}$ Dies wird vor allem darauf zurückgeführt, dass die menschenrechtliche Sorgfaltspflicht bislang nicht im Betriebsverfassungsgesetz (BetrVG) verankert ist und in Anbetracht der vielfältigen Aufgaben von Betriebsräten kaum Kapazitäten bestehen, das Thema zu ergründen. In den meisten Fällen betrachten Betriebsräte die Achtung der Menschenrechte als Managementaufgabe. Wenn es eine stärkere Befassung damit gibt, liegt dies oft an persönlichen Verbindungen oder einem Lieferketten-Skandal, der das eigene Unternehmen betrifft. Dabei zeigen Untersuchungen, dass Betriebsräte an einigen Schnittstellen von ihren Unternehmen eingebunden werden. Dies ist insbesondere bei der menschenrechtlichen Grundsatzerklärung der Fall, wenn ein Beschwerdemechanismus aufgesetzt wird oder Lieferantenaudits geplant und ausgewertet werden. Ein entsprechend ausgestaltetes Lieferkettengesetz könnte Belegschaft und Betriebsräte stärker für die Thematik sensibilisieren, eine Beteiligung bezüglich der Handlungsstrategien des Unternehmens vorsehen und die Kooperation mit den betreffenden ausländischen Arbeitnehmervertretungen fördern.

\subsection{Starke Durchsetzungsmechanismen verankern} Entsprechend den UN-Leitprinzipien muss es vorrangiges Ziel eines Lieferkettengesetzes sein, Menschenrechtsver- letzungen in GWK vorzubeugen. Dies wiederum setzt voraus, dass Unternehmen die menschenrechtliche Sorgfalt tatsächlich in ihre Organisationsabläufe integrieren und bei ihren Geschäftspraktiken berücksichtigen. Ein Lieferkettengesetz, das mit der menschenrechtlichen Sorgfaltspflicht gegenüber Unternehmen ein Gebot statuiert, muss dieses wirksam absichern.

In der Breite eignet sich hierfür eine ordnungsrechtliche behördliche Prüfung. In Frankreich, wo fehlende menschenrechtliche Sorgfalt von Unternehmen nach der branchenunabhängigen Loi de vigilence von 2017 nur gerichtlich - und damit einzelfallbezogen - geltend gemacht werden kann, wird derzeit erwogen, eine behördliche Überwachung einzuführen, um regelmäßig zu überblicken, ob sich die Unternehmen im Anwendungsbereich compliant verhalten. Eine entsprechende Berichtspflicht sollte die Kontrolle vergleichbar machen und vereinfachen. Erfahrungen mit dem französischen Gesetz zeigen, dass die Behörde nicht ausschließlich die Erfüllung der Berichtspflicht überwachen soll. Die Prüfung der Behörde sollte alle Elemente der Sorgfaltspflicht erfassen. Diese Aufgabe kann allerdings nur effektiv ausgeübt werden, wenn eine fachlich geeignete Behörde ausreichend ausgestattet wird und ermächtigt ist, bei Verstößen Bußgelder zu verhängen.

Auswertungen, die den UK Modern Slavery Act mit dem UK Bribery Act vergleichen, zeigen, dass ersterer, der nur mit Berichtspflicht umgesetzt wird, wenig Auswirkung auf Unternehmenspolitik und -verhalten zur Vermeidung moderner Formen der Sklaverei zeigte. Anders verhält es sich bezüglich der Korruptionsprävention erfasster Unternehmen bei letzterem Gesetz, das strafrechtlich und mit Bußgeldern durchgesetzt wird. ${ }^{6}$ Für erhebliche Menschenrechtsverstöße sollte ein Lieferkettengesetz deshalb eine zivilrechtliche Haftung vorsehen. Diese Sanktionierungsmöglichkeit wird zwar in den UN-Leitprinzipien nur als eine mögliche Ausformung der Achtungspflicht erwähnt. Sie hätte aber voraussichtlich mehrere wünschenswerte Effekte. Sie würde als finanzielles Risiko für Unternehmen dazu führen, dass die Thematik menschenrechtlicher Sorgfalt im Unternehmen aufgewertet würde, und statt oder neben etwa der Sustainability-Abteilung würde nun auch die Compliance-Einheit damit befasst. Zusätzlich schafft die zivilrechtliche Haftung eine Grundlage,

4 Konkretisierungen finden sich in den sektorspezifischen Leitfäden der OECD.

5 Hadwiger, F./ Hamm, B. / Vitols, K. / Wilke, P. (2017): Menschenrechte im Unternehmen durchsetzen. Internationale Arbeitnehmerrechte: Die UN-Leitprinzipien als Hebel für Betriebsräte und Gewerkschaften, Bielefeld.

6 LeBaron, G. / Rühmkorf, A. (2017): Steering CSR Through Home State Regulation: A Comparison of the Impact of the UK Bribery Act and Modern Slavery Act on Global Supply Chain Governance, in: Global Policy 8 (Suppl. 3), S. 15-28. 
geschädigten Arbeitnehmern und Gemeinden Zugang zu Recht zu ermöglichen und die Wiedergutmachung von Rechtsgutverletzungen wahrscheinlicher zu machen. Eine verschuldensabhängige, deliktische Haftung, die an Vorhersehbarkeit und Vermeidbarkeit anknüpft, ließe sich in das bestehende deutsche Deliktsrecht einpassen.

Um die Rechtsverfolgung wirksamer zu machen, sollten eine Beweislastumkehr, eine widerlegliche Verschuldensvermutung oder ein Anscheinsbeweis berücksichtigt werden, da Geschädigte im Normalfall Anspruchsvoraussetzungen nicht darlegen und beweisen können, die sich in der Sphäre der Unternehmen abspielen. Prozessual sind daneben Formen der Prozessstandschaft durch Gewerkschaften oder ausgewiesene NRO interessant. Andererseits erwarten auch Unternehmen zu Recht Rechtsicherheit zu den Grenzen ihrer Einstandspflicht. Eine wichtige Begrenzung gibt bereits die menschenrechtliche Sorgfaltspflicht selbst vor. Zwar sollte sie für die ganze Lieferkette gelten, der Pflichtenumfang muss aber für Unternehmen verhältnismäßig sein. Deshalb richtet sich die Komplexität des Verfahrens aus Risikoermittlung, Folgemaßnahmen und Wirksamkeitskontrolle nach der Größe des Unternehmens, der Art seiner Geschäftstätigkeit und seines Einflusses auf den Tatbeitrag sowie nach Wahrscheinlichkeit, Schwere, Reparabilität, Vorhersehbarkeit und Vermeidbarkeit menschenrechtlicher Beeinträchtigungen.

Denkbar ist auch, dass Unternehmen ihr Haftungsrisiko reduzieren, wenn sie ihre Sorgfaltspflicht in gemeinsamen Brancheninitiativen oder Multi-Stakeholder-Initiativen (MSI) erfüllen. Dies könnte sogar dazu führen, dass das Ambitionsniveau solcher Standards steigt. Wesentlich sind hierbei die Anforderungen an solche branchenspezifischen MSI-Standards. Sie müssen zum einen die Bestandteile der gesetzlichen Sorgfaltspflicht abbilden, was besonders für die einzelfallbezogenen Abhilfemaßnahmen schwer vorstellbar bleibt. Zum anderen müssen die Governance-Strukturen Unabhängigkeit, Konfliktlösung und Ausschlussmöglichkeiten von nicht erfüllenden Mitgliedern, Transparenz und Teilhabe lokaler Stakeholder garantieren.

\subsection{Akzeptanz durch Umsetzbarkeit erhöhen}

Die Akzeptanz der vom Gesetz erfassten Unternehmen wäre für die Implementierung hilfreich. Hierfür muss sich die menschenrechtliche Sorgfaltspflicht intern gut umsetzen lassen.

Mannigfache staatliche, private und zivilgesellschaftliche Leitfäden illustrieren Beispiele und Einzelschritte der unternehmensinternen Integration der UN-Leitprinzipien. ${ }^{7}$ Die schon bisher gepflegte Zusammenarbeit von Unternehmen in Brancheninitiativen o. Ä. kann die Vorbereitungs- und Einführungsphase erleichtern. Es ist sogar zu erwarten, dass Unternehmen aufgrund einer allgemeinen gesetzlichen Regelung ihre Prozesse erneuern und innovativ verbessern. Hierfür spricht auch der Fortschritt der Digitalisierung, die es beispielsweise vereinfacht, den
Weg einzelner Produkt(teile) abzubilden. Wer seine komplexen - Lieferketten kennt, entdeckt voraussichtlich schneller über den Menschenrechtsaspekt hinausgehende Unternehmensrisiken und kann hierauf reagieren.

Viele deutsche Unternehmen haben bereits Erfahrung, Sorgfaltspflichten in Unternehmensprozesse zu übersetzen. Sie müssen sich schon jetzt mit den bestehenden Vorschriften, etwa der französischen Loi de vigilence, befassen. Einige setzen bereits seit fast zehn Jahren die Sorgfaltspflichten beim Import von Konfliktmineralien aus der Kongo-Region nach dem amerikanischen Dodd Frank Act um. Machbar erscheint menschenrechtliche Sorgfalt auch vor dem Hintergrund, dass der erforderliche Risikoanalyse-Abhilfe-Monitoring-Kommunikationsprozess auf Verfahren des herkömmlichen Risikomanagements aufsetzt bzw. auf Plan-Do-Check-Act Zyklen des Compliance Managements, wie etwa bei der Korruptionsprävention. ${ }^{8}$

Im Sinne besserer Umsetzbarkeit für Unternehmen haben diese dafür argumentiert, die menschenrechtliche Sorgfalt auf die erste Zuliefererstufe (tier 1) zu beschränken. Wo die Risiken in GWK auftreten, hängt stark von der Branche und der Art der GWK ab (vgl. dazu die Beiträge von Teipen/Mehl und Marslev et al. in diesem Heft). Mit tier 1 wären gegebenenfalls ausschließlich große Importgesellschaften erfasst oder - wie in der Automobilindustrie einheimische Zulieferer. Kinderarbeit etwa findet jedoch bereits bei der Rohstoffgewinnung statt, beim Abholzen von Feldern für Kakaoanbau oder bei der Baumwollernte. Eine „pragmatische“ Verkürzung der Risikoanalyse sowie der Abhilfe- und Wiedergutmachungsmaßnahmen auf tier-1-Stufe liefe dem Sinn der Gesetzgebung entscheidend zuwider und würde Unternehmen sogar Anreize liefern, eine für die globale Arbeitsteilung nicht unbedingt erforderliche, aber menschenrechtlich ungefährliche tier-1-Stufe einzubauen.

\subsection{Level playing field schaffen}

Bislang nehmen diejenigen deutschen Unternehmen, die sich für soziale Nachhaltigkeit in der Lieferkette engagieren, damit verbundenen Aufwand in Kauf, während andere von der Regelungslücke profitieren und „unter dem

7 Vgl. z.B. den OECD-Leitfaden für die Erfüllung der Sorgfaltspflicht für verantwortungsvolles unternehmerisches Handeln, https://mneguidelines.oecd.org; BMU (Bundesministerium für Umwelt, Naturschutz, Bau und Reaktorsicherheit) (2017): Schritt für Schritt zum nachhaltigen Lieferkettenmanagement - Praxisleitfaden für Unternehmen, Berlin; Shift/MAZARS (2015): Berichtsrahmen für die UN-Leitprinzipien für Wirtschaft und Menschenrechte mit Umsetzungshinweisen, https://www.ungpreporting.org/ wp-content/uploads/UNGPRF_Deutsch_Dez2017.pdf

8 Vgl. exemplarisch anhand von drei Fallstudien Müller-Hoff, C./ Saage-Maaß, M. (2019): Menschenrechte vor Profit: So wird's gemacht - Fallstudien zur Machbarkeit menschenrechtlicher Sorgfalt im Unternehmen, hrsg. v. European Center for Constitutional and Human Rights et al., Berlin. 
Radar fliegen“. Solche Wettbewerbsverzerrungen könnte ein Gesetz abbauen. Dafür müssen die Anforderungen an seinen Anwendungsbereich entsprechend ausgerichtet werden. Es muss eine kritische Menge an Unternehmen erreichen. Der Schwellenwert des NAP für Unternehmen mit mehr als 500 Arbeitnehmern erreicht ca. 7200 Unternehmen und ist in der deutschen Unternehmenslandschaft inzwischen bekannt. Andererseits gehen die UN-Leitprinzipien davon aus, dass alle Unternehmen ungeachtet ihrer Größe menschenrechtliche Sorgfalt walten lassen können. An einem stark mittelständisch geprägten Wirtschaftsstandort wie Deutschland sind eben auch diese Firmen in GWK integriert und sollten erfasst werden, sofern sie in sehr menschenrechtsriskanten Branchen oder Gegenden tätig sind. Die derzeitigen Vorschläge aus dem Umfeld des BMWi, die ca. 300 deutschen Unternehmen mit mehr als 5000 Beschäftigten zu erfassen, hätten nur wenig Effekt, ein einheimisches level playing field für soziale Nachhaltigkeit zu schaffen. Aus demselben Grund wäre es sinnvoll, ausländische Unternehmen mit Geschäftstätigkeit in Deutschland ebenfalls zu erfassen.

\section{Herausforderungen für wirksames soziales Upgrading}

Selbst ein in sich schlüssiges, anspruchsvolles deutsches Lieferkettengesetz begegnet Rahmenbedingungen, die seiner Wirksamkeit und realem sozialen Upgrading hinderlich sind.

Fehlende internationale Governance: Die Governance angemessener Arbeitsbedingungen weltweit bleibt lückenhaft. Trotz der nationalen gesetzgeberischen Vorstöße, einer entsprechenden Initiative auf EU-Ebene und eines UN-Treaty-Prozesses fehlen bindende internationale Abkommen zu GWK. Auch die UN-Leitprinzipien füllen diese Lücke nicht. Trotz ihrer transnational harmonisierenden Wirkung sind sie grundsätzlich als rechtlich unverbindliches, völkerrechtliches „soft law“ einzuordnen. Der Umfang ihrer Transferwirkung auf nationale Ebene bleibt unberechenbar. Über die Erwartung des Menschenrechtsrats der UN hinaus, dass Staaten in nationalen Aktionsplänen Handlungsbedarf gegenüber ihren Unternehmen ermitteln, stellen die UN-Leitprinzipien keine Mittel zu Verfügung, sofern Regierungen der politische Wille zu einem solchen Prozess fehlt.

Fehlende Anreize für Regierungen des globalen Südens: Die UN-Leitprinzipien differenzieren nicht innerhalb der ersten Säule staatlicher Schutzpflichten für Menschen-

9 Dünhaupt, P./ Herr, H./ Mehl, F. / Teipen, C: (2019): Entwicklungschancen durch Integration in globale Wertschöpfungsketten: ein Länder- und Branchenvergleich, in: WSI-Mitteilungen 72 (5), S. 403-411, https://www.wsi.de/de/ wsi-mitteilungen-entwicklungschancen-durch-integrationin-globale-wertschoepfungsketten-ein-laender-und-18858. htm; vgl. auch den Beitrag von Teipen/Mehl in diesem Heft. rechte. Trotzdem unterscheiden sich die Ausgangslagen der angesprochenen Staaten zum Teil grundlegend. Viele Produktionsländer halten - in Corona-Zeiten umso mehr - ihre politökonomischen Interessen als attraktiver Wirtschaftsstandort für unvereinbar mit konsequenter Umsetzung von Arbeits- und Menschenrechtsstandards. Hier setzt auch ein nationales Lieferkettengesetz nur mittelbare Anreize. Stärkere Anreize, etwa Mindestlöhne regelmäßig zu erhöhen, müssten wohl handelsrechtlich unterlegt werden.

Fehlende Politikkohärenz: Investitionsabkommen und Handelspolitik berücksichtigen das Anliegen, soziale Faktoren in GWK zu verbessern, oft nicht ausreichend. Unternehmen mit Außenwirtschaftsförderung und Teilnahme an öffentlicher Beschaffung zu unterstützen, die ihre menschenrechtliche Sorgfaltspflicht nach obigem Vorbild erfüllen, wäre hier ein Ansatz zu mehr Politikkohärenz.

Schwache industrielle Beziehungen: Es wird argumentiert, dass soziales Upgrading u. a. starke Gewerkschaften voraussetze. ${ }^{9}$ Nationale Regulierungen, die die Vorgaben der UN-Leitprinzipien hierzu beherzigen, können in begrenztem Umfang dazu beitragen, dass die Rolle der Gewerkschaften gestärkt wird, kaum aber den fehlenden institutionellen Rahmen ersetzen.

\section{Ausblick}

Menschenrechtsverletzungen in Lieferketten finden häufig in einem komplexen Umfeld statt. Insofern wird ein deutsches, selbst europäisches Lieferkettengesetz nicht alle menschenrechtlichen Missstände beseitigen. Hierzu müssten auch die Regierungen der produzierenden Länder durch Positivlisten bzw. die Handelspolitik oder andere Instrumente stärkere Anreize haben, sich initiativ für Verbesserungen in ihren Ländern einzusetzen. Gleichwohl scheint der Moment für einen Anfang gut gewählt. Es wäre zu begrüßen, wenn der Schwung des Entwicklungsund des Arbeitsministers noch in dieser Legislaturperiode zu einem qualitätsvollen deutschen Lieferkettengesetz führen und zu einem Auftakt für eine europäische Regelung beitragen würde. Denn schließlich sollte in einem Rechtsstaat der Schutz hochrangiger Rechtsgüter wie der von Menschenrechten nicht dauerhaft der Freiwilligkeit überlassen bleiben.

\section{AUTORIN}

STEFANIE LORENZEN, Prof. Dr., ist Professorin für Arbeitsrecht an der Hochschule für Wirtschaft und Recht Berlin und Mitglied im Beirat des staatlichen Textilsiegels "Grüner Knopf". Forschungsschwerpunkte: Mitbestimmung, internationales Arbeitsrecht und Regulierung transnationaler Wertschöpfungsketten.

stefanie.Iorenzen@hwr-berlin.de 